\title{
Inoculação micorrízica no crescimento de mudas de angico em solo de cerrado
}

\author{
Maria Aparecida Alves Sugai $\left({ }^{1}\right)$, Leonardo Santos Collier $\left(2^{*}\right)$, Orivaldo José Saggin-Júnior $\left({ }^{3}\right)$ \\ (') Universidade Federal do Tocantins, Programa de Pós-graduação em Ciências do Ambiente, Q. 109 Norte, Av. NS 15, \\ Bloco III, 77001-090 Palmas (TO). \\ (2) Universidade Federal de Goiás, Escola de Agronomia e Engenharia de Alimentos, Caixa Postal 131, 74001-970 Goiânia (GO). \\ (3) Embrapa Agrobiologia, Caixa Postal 74505, 23890-000 Seropédica (RJ). \\ (*) Autor correspondente: leouft@gmail.edu.br
}

Recebido: 12/jun./2010; Aceito: 14/out./2010.

Resumo

O objetivo desse estudo foi avaliar o crescimento de mudas de Angico [Anadenanthera macrocarpa (Benth.) Brenan] sob o efeito da inoculação de fungos micorrízicos arbusculares (FMAs) em solo preservado e antropizado. O experimento foi desenvolvido em casa de vegetação, no período de janeiro a setembro de 2006 . O arranjo experimental foi em delineamento inteiramente casualizado, em esquema fatorial de $2 \times 4$, sendo dois tipos de solo (preservado ou antropizado) em quatro tratamentos distintos de inoculação micorrízica sendo (1) Solo esterilizado submetido à inoculação com a mistura Glomus etunicatum + Paraglomus brasilianum; (2) Solo esterilizado submetido à inoculação com a mistura Glomus etunicatum + Gigaspora margarita; (3) Solo esterilizado não inoculado (controle) (4) Solo natural (não esterilizado e não inoculado), com cinco repetições. Foram avaliados o crescimento em altura a cada 15 dias e, aos 150 dias, a área foliar, matéria seca da parte aérea e das raízes, comprimento de raízes, teores de nutrientes da parte aérea e colonização micorrízica. Melhor crescimento das mudas de angico foi observado no tratamento com solo esterilizado e inoculado com a mistura de G. margarita e G. etunicatum, o qual refletiu em aumento da área foliar, matéria seca da parte aérea e das raízes, assim como maior comprimento de raízes e colonização micorrízica, tanto no solo da área preservada quanto no da antropizada.

Palavras-chave: Anadenanthera macrocarpa, micorrização, área degradada, leguminosa arbórea.

\section{Mycorrhizal inoculation on angico seedlings growing with soil from cerrado}

\section{Abstract}

The objective of this research was to evaluate the Angico seedling growth [Anadenanthera macrocarpa (Benth.) Brenan] under the effect of arbuscular mycorrhizal fungi inoculation (FMAs) in preserved and antropized soils. This study was developed under greenhouse condition and the experiment was arranged in a complete random design within a factorial scheme $2 \times 4$ with five replications. There were two soils (preserved and antropized) and four treatments of mycorrhizal inoculation: (1) sterilized soil and inoculated with association of Glomus etunicatum + Paraglomus brasilianum; (2) sterilized soil and inoculated with association of Glomus etunicatum + Gigaspora margarita soil; (3) sterilized and not inoculated soil, i.e., control; and (4) natural soil (not sterilized and not inoculated). The growth in height was evaluated each 15 days and the leaf area, root and shoot dry matter, root length, shoot nutrient contents and mycorrhizal colonization were evaluated on the $150^{\text {th }}$ day. The best growth of Angico seedlings was observed in the treatment with sterilized soil inoculated with a mixture of Gigaspora margarita and Glomus etunicatum, which caused increases in leaf area, root and shoot dry matter, as well as longer roots and larger mycorrhizal colonization, in both preserved and antropized soils.

Key words: Anadenanthera macrocarpa, mycorrhization, degraded area, arboreal plants. 


\section{INTRODUÇÃO}

O desmatamento das florestas no Brasil vem trazendo consequências ecológicas e ambientais bastante visíveis, prejudicando as áreas de nascentes de rios, ao aumentar a exposição do solo, dificultando a regeneração e oferecendo oportunidades para estabelecimento de espécies invasoras. A retirada da vegetação de mata reflete no potencial de regeneração do solo, e determinadas espécies florestais possuem limitaçôes de restabelecimento em solo degradado que podem ser atribuídas à aquisição dos nutrientes, os quais muitas vezes estão indisponíveis nesses solos. Na recuperação dessas áreas degradadas é necessário aplicar estratégias que possam aproximar as características da floresta anteriormente existentes, de forma rápida e permanente.

Uma das alternativas para diminuir a inserção de insumos no reflorestamento de um solo degradado é propiciar melhor absorção dos nutrientes naturais pelas plantas. Os microrganismos do solo promotores de crescimento de plantas podem ser alternativa ao uso de fertilizantes químicos que, muitas vezes, onera ou inviabiliza a produção das mudas (SANTOS et al., 2008). Nesse contexto, os microrganismos simbióticos, tais como os fungos micorrízicos arbusculares (FMAs), exercem papel fundamental na manutenção dos sistemas florestais, podendo auxiliar na absorção dos nutrientes do solo e influenciar diretamente no crescimento das plantas.

Os FMAs associam-se à maioria das espécies de plantas e são capazes de absorver nutrientes do solo que são transferidos para as raízes do hospedeiro (WANG e QIU, 2006). Atuam como um complemento do sistema radicular da planta hospedeira capaz de aumentar a absorção de $\mathrm{P}$ e outros nutrientes, proteção contra patógenos e maior resistência ao estresse hídrico (Lynch e Ho, 2005; ColozZI Filho e Cardoso, 2000), dessa forma, favorecendo o crescimento e antecipando o plantio de mudas em campo, promovendo maior sobrevivência das mudas em viveiros e após o transplantio no campo e nos períodos secos (VANDresen et al., 2007; Miranda e Miranda, 2001).

O conhecimento das espécies arbóreas nativas com bom potencial de resposta à inoculação com FMAs é de grande importância para a recuperação de áreas degradadas e florestamento de espécies economicamente importantes, uma vez que na maioria das regióes destinadas ao reflorestamento há limitaçóes nutricionais, podendo dificultar o estabelecimento inicial das mudas no solo.

A espécie nativa Anadenanthera macrocarpa (Benth) Brenan (Angico) é uma leguminosa arbórea economicamente importante, de crescimento rápido e muito utilizada para reflorestamentos de áreas degradadas ou de preservação permanente (Pagano et al., 2008; SANTOS et al., 2008; LorenzI, 2000). A associação com FMAs em campo e nas mudas submetidas à inoculação com esses fungos na presença de fertilizantes tem sido constatada por alguns autores (Pereira et al., 1996; CARneiro et al., 1998; Gross et al., 2004). Porém o desenvolvimento dessa espécie micorrizada e sem adição de nutriente em casa de vegetação ainda é pouco explorado.

O objetivo deste estudo foi avaliar o crescimento de mudas de angico sob efeito de diferentes tratamentos de inoculação com fungos micorrízicos arbusculares em solo de Cerrado preservado e em solo de Cerrado antropizado.

\section{MATERIAL E MÉTODOS}

O experimento foi desenvolvido em casa de vegetação em Gurupi, Tocantins, localizado a $11^{\circ} 43^{\prime} 45^{\prime \prime}$ de latitude Sul e $49^{\circ} 04^{\prime} 07^{\prime \prime}$ longitude Oeste, nos períodos de janeiro a setembro de 2006. As mudas de angico foram formadas em substrato oriundo de um Latossolo Vermelho eutrófico coletado no período chuvoso na camada de $0-20 \mathrm{~cm}$ em uma área de preservaçáo permanente (solo preservado) no assentamento rural Vale-Verde e de um Latossolo Vermelho-Amarelo distrófico em uma área antropizada de pastagem (solo antropizado), ambos localizados no município de Gurupi (TO).

De acordo com a análise, estes solos possuem as seguintes características químicas, respectivamente, na área preservada e na antropizada: $\mathrm{MO}$ (Walkley-Black) 22 e $16 \mathrm{~g} \mathrm{dm}^{3} ; \mathrm{pH} 5$ e $4,7 \mathrm{em} \mathrm{CaCl}_{2} ; \mathrm{Al}^{+3} 1$ e $5,1 \mathrm{mmol}_{c}$ $\mathrm{dm}^{-3} \mathrm{em} \mathrm{KCl}$; P 3,4 e $2 \mathrm{mg} \mathrm{dm}^{-3}$; K 22,8 e 24,7 $\mathrm{mg} \mathrm{dm}^{-3}$ em Mehlich I; $\mathrm{Mg}^{+2}$ 11,3 e $16 \mathrm{mmol}_{\mathrm{c}} \mathrm{dm}^{-3} ; \mathrm{Ca}^{+2} 5,5$ e $10,5 \mathrm{mmol}_{\mathrm{c}} \mathrm{dm}^{-3} \mathrm{e} \mathrm{H}+\mathrm{Al}$ extraídos por acetato de cálcio 15 e $29 \mathrm{mmol}_{\mathrm{c}} \mathrm{dm}^{-3}$ (EMBrapa, 1997). Os substratos foram autoclavados em recipiente metálico com capacidade de 5 a 7 quilogramas na temperatura de $121^{\circ} \mathrm{C}$ e pressão de $1,5 \mathrm{~atm}$ duas vezes por uma hora e intervalo de um dia, de acordo com a metodologia adotada por MARINHO et al. (2004). A intenção da esterilização em autoclave foi eliminar os fungos micorrízicos nativos e outros microrganismos presentes no solo para inoculação da espécie desejada.

Para aumentar o índice de germinação do angico realizaram-se a escarificação e a embebição das sementes em água por 24 horas antes do semeio (BRASIL, 1992; SCALON et al., 2005). Em seguida, as sementes foram germinadas em sacos plásticos de polietileno com capacidade para 3 quilogramas de solo. Após a germinação, as mudas foram submetidas à inoculação com 10 gramas de solo-inóculo contendo esporos e fragmentos de raízes colonizadas pelas espécies Glomus etunicatum, Paraglomus brasilianum e Gigaspora margarita, provenientes de vaso de multiplicação, fornecido pela Embrapa Cerrados e Agrobiologia. O inoculante foi colocado a $3 \mathrm{~cm}$ aproximadamente, abaixo da superfície do solo contido no recipiente.

Utilizou-se esquema fatorial $(2 \times 4)$, sendo dois tipos de solo (preservado ou antropizado) e quatro tratamentos de inoculação (solo esterilizado inoculado com Glomus etunicatum + Paraglomus brasilianum; esterilizado 
inoculado com Glomus etunicatum + Gigaspora margari$t a$; esterilizado não inoculado e natural (não esterilizado e não inoculado), distribuídos em um delineamento inteiramente casualizado com cinco repetições, totalizando 40 plantas. As mudas foram preservadas em condiçóes de casa de vegetação e irrigadas periodicamente, para manter a umidade do solo na capacidade de campo.

O crescimento em altura $(\mathrm{cm})$ foi acompanhado com régua graduada a cada 15 dias a partir do colo até o meristema apical da parte aérea. Após 150 dias do plantio, as plantas foram colhidas inteiras para avaliação das seguintes variáveis: área foliar, através do medidor de área foliar marca CI - 202 Area Meter, com distribuição uniformizada de todas as folhas na prancha, sendo os resultados expressos $\mathrm{em} \mathrm{cm}^{2}$; massa seca da parte aérea e das raízes após secagem em estufa a $65{ }^{\circ} \mathrm{C}$ até massa constante e medida em balança semianalítica de acordo com método adotado por SENA et al. (2004); comprimento das raízes pivotantes inteiras, coletadas cuidadosamente e medidas com régua graduada, da base do caule até o meristema apical da raiz; análises dos teores dos macronutrientes $\mathrm{N}$, $\mathrm{P}, \mathrm{K}, \mathrm{Ca}, \mathrm{Mg}$ e $\mathrm{S}$ na parte aérea, após esta ter sido lavada em água corrente e, seca em estufa a $65^{\circ} \mathrm{C}$ e triturada em moinho de facas (Malavolta et al., 1997).

Para avaliação da porcentagem de colonização micorrízica foram coletadas amostras de um grama de raízes frescas e finas; cada planta teve três amostras dessa natureza para cada uma das cinco repetiçóes. As raízes foram lavadas com água corrente, diafanizadas com $\mathrm{KOH} 10 \%$ por aproximadamente 12 horas em temperatura ambiente, aquecidas por 40 minutos a $90^{\circ} \mathrm{C}$, em seguida, acidificadas com $\mathrm{HCl}$ $2 \%$ e coradas com solução de azul de tripano a 0,05\% em soluçáo de lactoglicerol (Philips e Hayman, 1970). A taxa de colonizaçáo foi determinada através da presença de estruturas fúngicas no tecido cortical a partir da análise visual de 50 fragmentos (totalizando 150 fragmentos em cada repetição) em lâminas com auxílio de microscópio óptico (Giovanetti e Mosse, 1980). Considerou-se raiz colonizada, todos os fragmentos nos tratamentos inoculados ou não, que continham pelo menos uma estrutura micorrízica. Devido à distribuiçáo normal desse tipo de atributo mensurado náo houve necessidade de transformação de dados através dos programas estatísticos.

Os dados obtidos foram submetidos à análise de variância e teste Tukey a 5\% de probabilidade utilizando-se o programa estatístico Sisvar (Ferreira, 1998).

\section{RESULTADOS E DISCUSSÃO}

Os dados do tratamento em solo esterilizado e não inoculado não são apresentados nos resultados deste trabalho devido à grande mortalidade das plantas nestas condiçóes em solos preservados e antropizados. Esta grande mortalidade indica que a espécie arbórea estudada é de elevada dependência micorrízica e que não consegue sobreviver em solos sem a simbiose micorrízica arbuscular, a não ser que seja aplicado fertilizante.

No solo preservado (Figura 1a) aos 150 dias após a germinação (DAG), o angico revelou melhor crescimento em altura quando submetido à inoculação com a mistura de Glomus etunicatum e Gigaspora margarita ou com os naturalmente presentes no solo do que com a mistura de G. etunicatum e Paraglomus brasilianum. Resultados semelhantes de melhor adaptação a determinado fungo foram observados por Rocha et al. (2006), em mudas de cedro, onde o crescimento vegetativo com os tratamentos Glomus clarum e Gigaspora margarita se destacou dos outros tratamentos de inoculação aos 180 dias após a germinação.

Alguns autores têm relatado benefícios com a inoculação de espécies de Gigaspora. O crescimento de mudas de maracujazeiro amarelo tem sido beneficiado com a inoculação de Gigaspora albida e G. margarita (CavalCante et al., 2002). SiLVA et al. (2004) verificaram diferenças na altura de maracujazeiro doce com a inoculação de G. albida aos 90 dias. Resposta significativa foi obtida também com inoculação de G. albida no crescimento de mangabeira (Costa et al., 2005). Saggin-Júnior e Siqueira (1995) ao considerar a área foliar do cafeeiro, observaram melhor
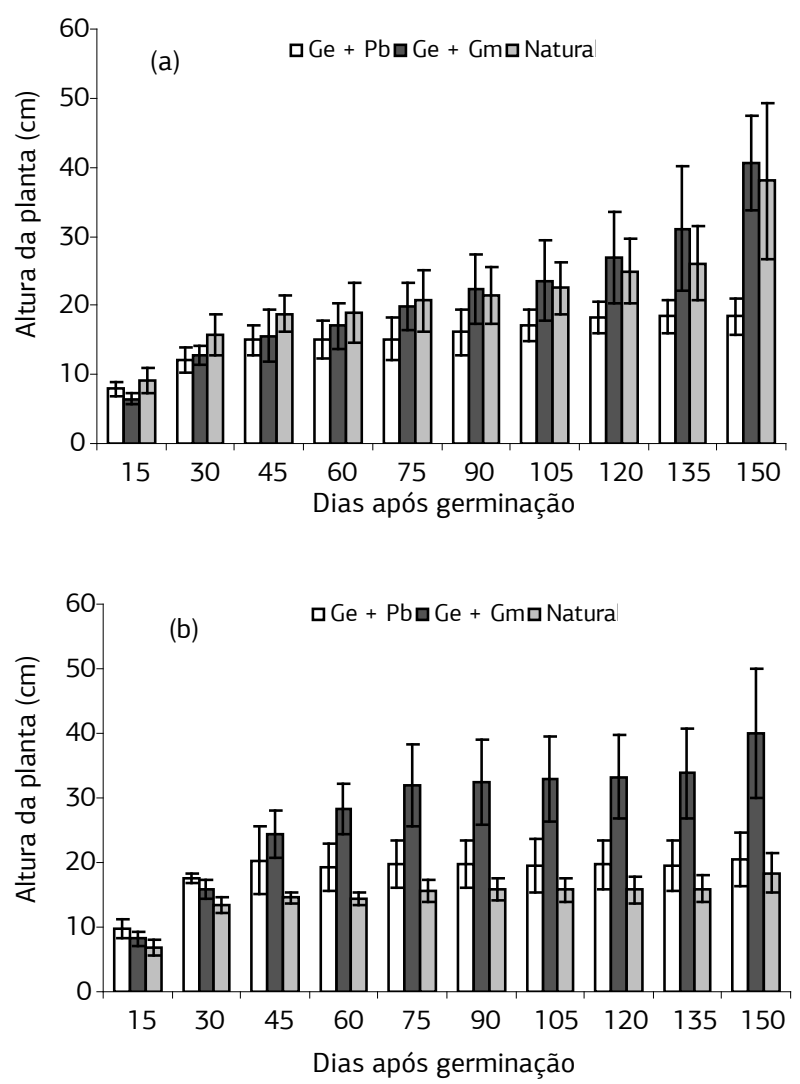

Figura 1. Altura de angico cultivado em solo preservado (a) e solo antropizado (b), esterilizado e submetido à inoculação com Glomus etunicatum $(\mathrm{Ge})$ + Paraglomus brasilianum $(\mathrm{Gb})$, Gigaspora margarita $(\mathrm{Gm})$ e em solo natural (não esterilizado e não inoculado). CV (\%) = 22,94 e 20,33 respectivamente. Dados não transformados. 
eficiência simbiótica no tratamento com $G$. margarita seguido de G. etunicatum aos 150 dias. Melhor efeito no crescimento de mudas de cafeeiro também foi observado em plantas colonizadas com a espécie G. margarita sobre o controle não inoculado (SAGgin-Júnior e Siqueira, 1996). Tristão et al. (2006), ao pesquisarem FMAs em mudas de cafeeiro, observaram maiores valores de produção de matéria seca da parte aérea, diâmetro do caule e altura em plantas colonizadas por G. margarita em solo com adição de substrato.

As plantas cultivadas em solo preservado natural tiveram melhor crescimento, do que as cultivadas em solo estéril submetido à inoculação com Glomus etunicatum + Paraglomus brasilianum (Figura 1a). Gross et al. (2004), estudando Anadenanthera peregrina, concluíram que em plantas cultivadas em solo de cerrado não autoclavado houve alta taxa de colonização fúngica nas raízes, influenciando positivamente nos teores $\mathrm{P}$ na planta. Essa ocorrência indica que a comunidade nativa de FMAs possui espécies eficientes em promover o crescimento desta arbórea.

No solo antropizado (Figura 1b) verifica-se aos 150 DAG que o tratamento Glomus etunicatum + Gigaspora margarita promoveu maior altura de plantas que os demais tratamentos. Neste solo, verifica-se que a comunidade nativa de FMAs (solo natural) náo foi tão eficiente quanto a do solo preservado. Possivelmente, alguma espécie de FMA eficiente para o angico foi eliminada com a antropização do solo ou a alteração de diversidade e dominância de espécies promovidas pela antropização, e esse processo tenha alterado a eficiência simbiótica da comunidade de FMAs para o angico.

Tendo em vista que o maior crescimento em altura do angico ocorreu nos tratamentos estéreis inoculados com Glomus etunicatum + Gigaspora margarita (Figura 1a,b) em ambos os solos e no tratamento com solo preservado natural (Figura 1a), acredita-se que $G$. margarita faça o diferencial entre os tratamentos de inoculação e que na comunidade de FMAs exista uma espécie tão eficiente para o angico quanto G. margarita. Além disso, Segundo Hartnet e Wilson (2002), a estrutura de algumas comunidades de plantas pode ser regulada simplesmente pela presença e abundância do fungo micorrízico e pelas combinaçôes mais eficientes entre espécies de plantas e FMAs co-existentes. Аввотt e Gazey (1994) relatam que pode ser vantajoso manter altos níveis de diversidade de espécies de FMAs no solo, independentemente de sua contribuição individual. Esse fato pode proporcionar maior oportunidade para que existam espécies de FMAs eficientes para a planta de interesse e que esta espécie predomine quando as condiçôes do solo forem modificadas (Miranda et al., 2005).

Apesar de ter ocorrido alta colonização das raízes de A. macrocarpa cultivada em solo antropizado, não foram constatadas diferenças significativas no tratamento com associação de Glomus etunicatum e Paraglomus brasilianum, quando comparado ao solo natural. Esse resultado talvez tenha sido influenciado pela ausência da diversidade de espécies do FMA nesse local e consequentemente menor aproveitamento dos nutrientes existentes no solo.

Quando cultivado em ambos os solos submetidos à inoculação com G. etunicatum e G. margarita ou no solo preservado natural, o angico foi beneficiado no desenvolvimento da área foliar, massa seca da parte aérea e de raízes (Tabela 1). Esses resultados comprovam a relaçáo no crescimento da parte aérea dessa espécie aos 150 DAG com os resultados de altura. A comunidade de FMAs nativa do solo preservado natural promoveu maior sistema radicular que ambos os tratamentos de inoculaçáo estudados, favorecendo o comprimento das raízes. Entre os tratamentos de inoculação, a mistura de G. etunicatum e $G$. margarita promoveu mais raízes do que G. etunicatum e P. brasilianum. No solo antropizado o resultado observado no sis-

Tabela 1. Área foliar (AF), matéria seca da parte aérea (MSPA), matéria seca das raízes (MSR) e comprimento de raízes (CR) de mudas de angico aos 150 dias após a germinação em solo preservado e antropizado

\begin{tabular}{|c|c|c|c|c|}
\hline \multirow[t]{3}{*}{ Tratamentos } & AF & MSPA & MSR & CR \\
\hline & $\mathrm{cm}^{2}$ & \multicolumn{2}{|c|}{$\mathbf{g ~ k g}^{1}$} & cm \\
\hline & \multicolumn{4}{|c|}{ Solo Preservado } \\
\hline $\mathrm{Ge}+\mathrm{Pb}$ & $2,87 b$ & $0,30 b$ & $0,45 b$ & $5,12 b$ \\
\hline $\mathrm{Ge}+\mathrm{Gm}$ & $11,03 a$ & $1,40 a$ & $1,61 \mathrm{a}$ & $6,32 \mathrm{a}$ \\
\hline Natural & $10,84 a$ & $1,42 \mathrm{a}$ & $1,44 a$ & $6,61 a$ \\
\hline \multirow[t]{2}{*}{ CV (\%) } & 32,22 & 32,13 & 32,81 & 9,60 \\
\hline & \multicolumn{4}{|c|}{ Solo Antropizado } \\
\hline $\mathrm{Ge}+\mathrm{Pb}$ & $3,04 c$ & $0,43 b$ & $0,70 b$ & $6,22 \mathrm{a}$ \\
\hline$G e+G m$ & $9,56 a$ & $1,40 a$ & $2,27 a$ & $6,30 a$ \\
\hline Natural & $7,69 a$ & $0,84 b$ & $1,07 b$ & $6,88 a$ \\
\hline CV (\%) & 28,91 & 33,25 & 26,54 & 8,79 \\
\hline
\end{tabular}

Médias seguidas da mesma letra na coluna năo diferem entre si pelo teste de Tukey a $5 \%$ de probabilidade. Solo submetido à inoculaçăo com Glomus etunicatum (Ge), Paraglomus brasilianum $(\mathrm{Pb})$, Gigaspora margarita $(\mathrm{Gm})$ e não esterilizado e não inoculado (Natural). Dados transformados - SQRT (Y). 
Tabela 2. Teores de macronutrientes na matéria seca da parte aérea da espécie Anadenanthera macrocarpa (angico) após150 dias da germinação em solo preservado ou antropizado submetidos a tratamento de esterilização e inoculação de FMAs

\begin{tabular}{|c|c|c|c|c|c|c|}
\hline \multirow[t]{3}{*}{ Tratamentos } & $\mathbf{N}$ & $\mathbf{P}$ & K & $\mathrm{Ca}$ & Mg & $\mathbf{S}$ \\
\hline & \multicolumn{6}{|c|}{$\mathbf{g ~ k g}^{1}$} \\
\hline & \multicolumn{6}{|c|}{ Solo Preservado } \\
\hline $\mathrm{Ge}+\mathrm{Pb}$ & $-(1)$ & - & - & - & - & - \\
\hline $\mathrm{Ge}+\mathrm{Gm}$ & $5,01 a$ & $0,79 a$ & $3,22 \mathrm{a}$ & $1,92 \mathrm{a}$ & $1,18 \mathrm{a}$ & $1,10 \mathrm{a}$ \\
\hline Natural & $4,43 a$ & $0,85 a$ & $3,10 a$ & $1,93 a$ & $1,27 a$ & $1,27 a$ \\
\hline \multirow[t]{2}{*}{ CV (\%) } & 3,90 & 6,68 & 4,56 & 7,77 & 4,19 & 6,32 \\
\hline & \multicolumn{6}{|c|}{ Solo Antropizado } \\
\hline $\mathrm{Ge}+\mathrm{Pb}$ & $-(1)$ & - & - & - & - & - \\
\hline $\mathrm{Ge}+\mathrm{Gm}$ & $4,18 b$ & $0,79 a$ & $2,94 b$ & $3,45 a$ & $1,24 \mathrm{a}$ & $1,29 a$ \\
\hline Natural & $5,20 a$ & $1,16 a$ & $4,58 a$ & $3,78 a$ & $1,57 a$ & $1,65 \mathrm{a}$ \\
\hline CV (\%) & 6,99 & 38,72 & 38,38 & 33,20 & 31,43 & 42,91 \\
\hline
\end{tabular}

(') Material insuficiente para análise. Médias seguidas da mesma letra nas colunas nâo diferem entre si pelo teste de Tukey a $5 \%$ de probalidade. Solo submetido à inoculação com Glomus etunicatum (Ge), Paraglomus brasilianum (Pb), Gigaspora margarita $(\mathrm{Gm})$ e não esterilizado e não inoculado (Natural). Dados transformados - SQRT (Y).

tema radicular corrobora o observado na parte aérea, sendo a inoculação com $G$. etunicatum e $G$. margarita mais benéfica do que os demais tratamentos (Figura 1a,b). É interessante observar também nestas figuras, que no solo preservado (Figura 1a) a comunidade nativa é mais rápida que $G$. margarita em promover resposta em crescimento do angico. Embora a mistura G. etunicatum e G. margarita seja a mais eficiente nos dois solos estudados, sua eficiência em promover o crescimento do angico somente é demonstrada a partir de 45 a 60 DAG.

Caravaca et al. (2005) ao pesquisarem o desenvolvimento de arbustos em ecossistemas degradado na região mediterrânea, verificaram que as plantas submetidas à inoculaçáo com Glomus claroideum e ou com a mistura de FMA nativos tiveram maior altura, diâmetro basal, massa seca e comprimento de raízes. G. margarita com Scutellospora heterogama foi mais eficiente no crescimento de mudas de gravioleira em solo fumigado quando comparado com Entrophospora colombiana e Gigaspora sp. (CHU et al., 2001). Nesse contexto, os resultados indicam que a associação de G. margarita com outras espécies de FMA pode ter favorecido o desenvolvimento das mudas de angico em solo estéril.

Devido à pouca produção de massa seca da parte aérea, não foi possível avaliar os nutrientes das plantas no tratamento com G. etunicatum e P. brasilianum no solo preservado e antropizado (Tabela 2). Dentre os nutrientes avaliados, o nitrogênio promoveu maior quantidade na parte aérea do angico em todos os tratamentos. No solo preservado, nos dois tratamentos analisados não houve diferenças nos teores de $\mathrm{P}, \mathrm{K}, \mathrm{Ca}, \mathrm{Mg}$ e S. Resultados semelhantes também foram observados por CARNEIRO et al. (2004) em mudas de espécies nativas. Esses mesmos autores sugerem que o baixo efeito dos fatores estudados, nas características vegetativas da planta, deve-se ao fato do substrato utilizado ser rico em nutrientes, especialmente o fósforo.
Tabela 3. Colonização micorrízica das raízes de angico em solo preservado ou antropizado submetido à inoculação com Glomus etunicatum (Ge), Paraglomus brasilianum (Pb), Gigaspora margarita $(\mathrm{Gm})$ em solo esterilizado e em solo náo esterilizado e não inoculado (Natural).

\begin{tabular}{|ccc|}
\hline Tratamentos & \multicolumn{2}{c}{ Colonização \% } \\
& Solo Preservado & Solo Antropizado \\
\hline $\mathrm{Ge}+\mathrm{Pb}$ & $5,60 \mathrm{a}$ & $6,09 \mathrm{a}$ \\
\hline $\mathrm{Ge}+\mathrm{Gm}$ & $6,65 \mathrm{a}$ & $6,60 \mathrm{a}$ \\
\hline Natural & $5,40 \mathrm{a}$ & $6,21 \mathrm{a}$ \\
\hline $\mathrm{CV}(\%)$ & 9,95 & 8,06 \\
\hline
\end{tabular}

Médias seguidas da mesma letra nas colunas não diferem entre si pelo teste de Tukey a $5 \%$ de probalidade. Dados transformados - SQRT (Y).

No solo antropizado natural houve diferenças significativas em todos os teores de nutrientes nas plantas, quando comparado ao tratamento inoculado com $G$. margarita e G. etunicatum. Como este tratamento tendeu a proporcionar menor crescimento, este efeito é provavelmente um efeito de diluição, que ocorre devido às plantas micorrizadas eficientemente acumularem mais matéria seca em solo com pouca capacidade de fornecimento de nutrientes (Siqueira e Franco, 1988).

De forma geral, o angico proporcionou alta colonização de suas raízes ficando acima de 60\% (Tabela 3). A maior porcentagem de colonização foi observada no tratamento com G. etunicatum e G. margarita nas duas áreas de estudo, sendo entre $66,0 \%$ e $66,5 \%$, considerada alta. A alta colonização pode ter influenciado no diferencial de crescimento desse tratamento. $\mathrm{O}$ aumento no crescimento da planta é atribuído à capacidade de absorção de nutrientes do solo, principalmente aqueles que se movimentam por difusão, com baixa mobilidade, como o $\mathrm{P}$, Cu e $\mathrm{Zn}$ (Colozzi-Filho e Balota, 1994).

A ocorrência de colonização micorrízica nas raízes das Leguminosae-Mimosoideae tem sido bastante relatada por 

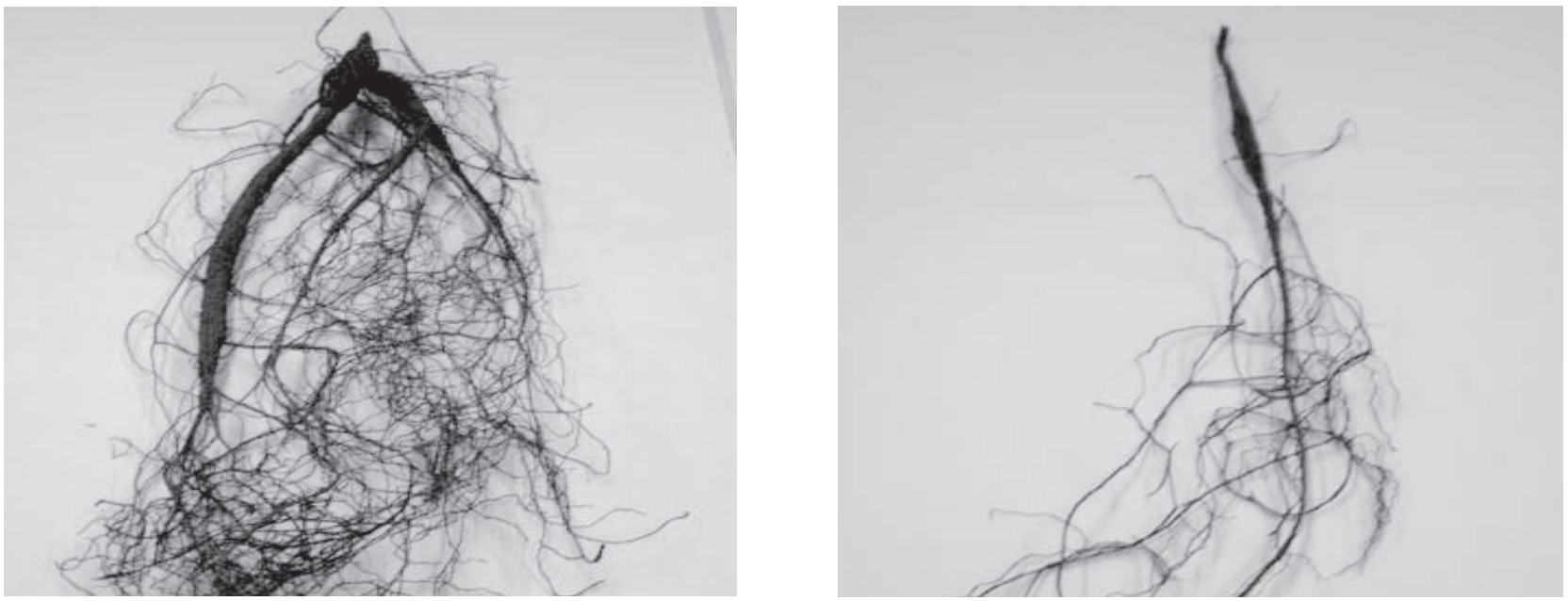

Figura 2. Aspecto do sistema radicular de mudas de Anadenanthera macrocarpa (angico) em solo antropizado estéril submetido à inoculação com Glomus etunicatum e Gigaspora margarita (a) e solo antropizado natural (b).

vários autores (CARNEIro et al., 1996; CARNEIro et al., 1998; Patreze e Cordeiro, 2005). Em espécies nativas cultivadas em viveiro e casa de vegetação, a colonização foi de média a alta, e as Leguminosae-Mimosoideae obtiveram índice de 86\% de micorrização (CARNEIro et al., 1998).

As estruturas observadas nas raízes do angico foram hifas intrarradiculares e extrarradiculares, vesículas, esporos e células auxiliares. A alta pigmentação observada na camada epidérmica desta espécie arbórea dificultou a identificação de arbúsculos, mesmo tendo permanecido por 12 horas em $\mathrm{KOH}(10 \%)$ na pré-descoloração.

Apesar de ter observado maior comprimento radicular no tratamento natural (sem inoculação) em ambosos tipos de solo (Tabela 1), o solo antropizado e submetido à inoculação com G. etunicatum e G. margarita propiciou aumento de volume no sistema radicular das mudas de angico quando comparadas ao solo natural (Figura 2). CARNEIRO et al. (2004) ao pesquisarem o desenvolvimento de mudas de espécie florestal submetidas à inoculação com FMA também observaram maior produção de raízes quando comparadas com as mudas náo inoculadas.

O aumento do volume e da extensão das raízes é de grande importância para mudas destinadas a reflorestamento em locais degradados ou de baixa fertilidade, podendo melhorar as condições de absorção de água e nutrientes e aumentar a sobrevivência no campo após o plantio. O processo de absorção de $\mathrm{P}$ é similar na hifa micorrízica e nas raízes, sendo muito influenciado pela extensão da hifa externa, bem como pelo requerimento de P da planta hospedeira (Rodrigues et al., 2003).

Trabalhos desenvolvidos por VANDRESEN et al. (2007) mostraram que mudas de angico submetidas à inoculação com FMAs tiveram maior sobrevivência após seis meses de crescimento no campo em relação às não-inoculadas. De acordo com Carneiro et al. (2004), as mudas de embaúba inoculadas tiveram maior sobrevivência ao estresse hídrico, principalmente quando associadas ao baixo teor de fósforo. Segundo Siqueira et al. (2002), além da alta capacidade e eficiência de absorçáo do $\mathrm{P}$, as hifas dos FMAs avançam solo adentro, absorvendo nutrientes fora da zona de esgotamento que é gerada em torno das raízes e transferida para a planta hospedeira nos arbúsculos.

\section{CONCLUSÃO}

Há alta colonizaçáo micorrízica nas raízes tanto nas plantas de angico cultivadas em solo preservado quanto no solo antropizado. A mistura de Glomus etunicatum e Gigaspora margarita é eficiente em promover benefícios no crescimento e na nutrição de Anadenanthera macrocarpa (angico), independentemente do tipo de solo.

\section{AGRADECIMENTOS}

Ao Conselho Nacional de Desenvolvimento Científico e Tecnológico - CNPq, pela bolsa de mestrado concedida à primeira autora. À Empresa Brasileira de Pesquisa Agropecuária - Embrapa, unidade Cerrado e Agrobiologia, pelos inoculantes fornecidos.

\section{REFERÊNCIAS}

ABBOTT, L.K.; GAZEY, C. An ecological view of the formation of VA mycorrhizas. Plant and Soil, v.159, p.69-78, 1994.

BRASIL. Ministério da Agricultura e Reforma Agrária. Regras para Análise de Sementes. Brasília, 1992. 365p.

CARAVACA, F.; ALGUACIL, M.M.; BAREA, J.M.; ROLDÁN, A. Survival of inocula and native AM fungi species associated with shrubs in a antropized Mediterranean ecosystem. Soil Biology and Biochemistry, v.37, p.227-233, 2005. 
CARNEIRO, M.A.C.; SIQUEIRA, J.O.; DAVIDE, A.C. Fósforo e inoculação com fungos micorrízicos arbusculares no estabelecimento de mudas de embaúba (Cecropia pachystachya Trec). Pesquisa Agropecuária Tropical, v.34, p.119-125, 2004.

CARNEIRO, M.A.C.; SIQUEIRA, J.O.; DAVIDE, A.C.; GOMES, L.J.; CURI, N.; VALE, F.R. DO. Fungo micorrízico e superfosfato no crescimento de espécies arbóreas tropicais. Scientia Forestalis, v.1, p.21-36, 1996.

CARNEIRO, M.A.C.; SIQUEIRA, J.O; MOREIRA, F.M.S.; CARVALHO, D. DE; BOTELHO, S.A.; SAGGIN JUNIOR, O.J. Micorriza arbuscular em espécies arbóreas e arbustivas nativas de ocorrência no sudeste do Brasil. Cerne, v.4, p.129-145, 1998.

CAVALCANTE, U.M.T.; MAIA, L.C.; COSTA, C.M.C.; CAVALCANTE, A.T.; SANTOS, V.F. Efeito de fungos micorrízicos arbusculares, da adubação fosfatada e da esterilização do solo no crescimento de mudas de maracujazeiro amarelo. Revista Brasileira de Ciência do Solo, v.26, p.1099-1106, 2002.

CHU, E.Y; MÖLlER, M. DE R.F.; CARVALHO, J.G. DE. Efeitos da inoculação micorrízica em mudas de gravioleira em solo fumigado e não fumigado. Pesquisa Agropecuária Brasileira, v.36, p.671-680, 2001.

COLOZZI-FILHO, A.; BALOTA, É.L. Micorrizas Arbusculares. In: HUNGRIA, M. ARAUJO, R.S. (Ed.). Manual de métodos empregados em estudos de microbiologia agrícola. Brasília: Embrapa, 1994. p.383-418.

COLOZZI-FILHO, A.; CARDOSO, E.J.B.N. Detecção de fungos micorrízicos arbusculares em raízes de cafeeiro e de crotalária cultivada na entrelinha. Pesquisa Agropecuária Brasileira, v.35, p.2033-2042, 2000.

COSTA, C.M.C.; CAVALCANTE, U.M.T.; GOTO, B.T.; SANTOS, V.F.; MAIA, L.C. Fungos micorrízicos arbusculares e adubação fosfatada em mudas de mangabeira. Pesquisa Agropecuária Brasileira, v.40, p.225-232, 2005.

EMBRAPA (Empresa Brasileira de Pesquisa Agropecuária). Centro Nacional de Pesquisa de Solos. Manual de métodos de análise de solo. 2.ed. Rio de Janeiro: Embrapa Solos, 1997. 212p.

FERREIRA, D.F. Sistemas de análise estatística para dados balanceados. Lavras: Ufla/DEX/Sisvar, 1998. 141p.

GIOVANETTI, M.; MOSSE, B. An evaluation of techniques for measuringvesicular-arbuscular mycorrhizal infection in roots. New Phytologist, v.84, p.489-500, 1980.

GROSS, E.; CORDEIRO, L.; CAETANO, F.H. Nodulação e micorrização em Anadenanthera peregrina VAR. falcata em solo de cerrado autoclavado e não autoclavado. Revista Brasileira de Ciência do Solo, v.28, p.95-101, 2004.

HARTNET, D.C.; WILSON, G.W.T. The role of mycorrhizas in plant community structure and dynamics: lessons from grasslands. Plant and Soil, v.244, p.319-331, 2002.

LORENZI, H. Árvores brasileiras: manual de identificação e cultivo de plantas arbóreas nativas do Brasil. 3.ed. São Paulo, 2000. 314p.
LYNCH, J.P.; HO, M.D. Rhizoeconomics: Carbon costs of phosphorus acquisition. Plant and Soil, v. 269, p.45-56, 2005.

MALAVOLTA, E.; VITTI, G.C.; OLIVEIRA, S.A. Avaliação do estado nutricional das plantas: princípios e aplicaçóes. 2.ed. São Paulo: Potafos, 1997. 319p.

MARINHO, N.F.; CAPRONI, A.L.; FRANCO, A.A.; BERBARA, R.L.L. Respostas de Acacia mangium Willd e Sclerolobium paniculatum Vogel a fungos micorrízicos arbusculares nativos provenientes de áreas degradadas pela mineração de bauxita na Amazônia. Acta Botânica Brasilica, v.18, p.141-149, 2004.

MIRANDA, J. C. C. DE; MIRANDA, L. N. Produção de mudas inoculadas com fungos micorrízicos arbusculares em viveiros. Brasília; Embrapa Cerrados, 2001. 2p. (Recomendação Técnica, n.24)

MIRANDA, J.C.C. DE; VILELA, L.; MIRANDA, L.N. Dinâmica e contribuição da micorriza arbuscular em sistemas de produção com rotação de culturas. Pesquisa Agropecuária Brasileira, v.40, p.1005-1014, 2005.

PAGANO, M.C.; CABELLO, M.N.; BELLOTE, A.F.; SÁ, N.M.; SCOTTI, M.R. Intercropping system of tropical leguminous species and Eucalyptus camaldulensis, inoculated with rhizobia and/or mycorrhizal fungi in semiarid Brazil. Agroforestry Systems, v.74, p.231-242, 2008.

PATREZE, C.M.; CORDEIRO, L. Nodulation, arbuscular mycorrhizal colonization and growth of some legumes native from Brazil. Acta Botanica Brasilica, v.19, p.527-537, 2005.

PEREIRA, E.G.; SIQUEIRA, J.O.; CURI, N.; MOREIRA, F.M.S.; PURCINO, A.Á.C. Efeitos da micorriza e do suprimento de fósforo na atividade enzimática e na resposta de espécies arbóreas ao nitrogênio. Revista Brasileira de Fisiologia Vegetal, v.8, p.59-65, 1996.

PHILIPS, J.M.; HAYMAN, D.S. Improved procedures for clearing roots and staining parasitic and vesicular - arbuscular mycorrhizal fungi for rapid assestment of infection. Transactions of the British Mycological Society, v.55, p.158-161, 1970.

ROCHA, F.S.; SAGGIN-JUNIOR, O.J.; SILVA, E.M.R. da; LIMA, W.L. DE. Dependência e resposta de mudas de cedro a fungos micorrízicos arbusculares. Pesquisa Agropecuária Brasileira, v.41, p.77-84, 2006

RODRIGUES, L.A.; MARTINS, M.A.; SALOMÃO, M.S.M.B. Uso de micorrizas e rizóbio em cultivo consorciado de eucalipto e sesbânia. II - absorção e eficiência de utilização de fósforo e fraçôes fosfatadas. Revista Brasileira de Ciência do Solo, v.27, p.593-599, 2003.

SAGGIN-JÚNIOR, O.J.; SIQUEIRA, J.O. Micorrizas arbusculares em cafeeiro. In: SIQUEIRA, J.O. (Ed.). Avanços em fundamentos e aplicação de micorrizas. Lavras: UFLA/DCS/DCF, 1996. p.203-254.

SAGGIN-JÚNIOR, O.J.; SIQUEIRA, J.O. Avaliação da eficiência simbiótica de fungos endomicorrízicos para o cafeeiro. Revista Brasileira de Ciência do Solo, v.19, p.221-228, 1995. 
SANTOS, D.R.; COSTA, M.C.S.; MIRANDA, J.R.; SANTOS, R.V.. Micorrizas e Rizóbio no crescimento e nutrição em $\mathrm{N}$ e $\mathrm{P}$ de mudas de angico-vermelho. Revista Caatinga, v.21, p.76-82, 2008 .

SCALON, S. DE P. Q.; MUSSURY, R.M.; WATHIER, F.; GOMES, A.A.; SILVA, K.A.; PIEREZAN, L.; SCALON FILHO, H. Armazenamento, germinação de sementes e crescimento inicial de mudas de Enterolobium contortisiliquum (Vell.) Morong. Acta Scientiarum Biological Sciences, v.27, p.107-112, 2005.

SENA, J.O.A.; LABATE, C.A.; CARDOSO, J.B.N. Caracterização Fisiológica da reduçáo de crescimento de mudas de citros micorrizadas em altas doses de fósforo. Revista Brasileira de Ciência do Solo, Viçosa, v.28, p.827-832, 2004.

SILVA, M.A. DA; CAVALCANTE, U.M.T.; SILVA, F.S.B. DA; SOARES, S.A.G.; MAIA, L.C. Crescimento de mudas de maracujazeiro-doce (Passiflora alata Curtis) associadas a fungos micorrízicos arbusculares (Glomeromycota). Acta Botânica Brasilica, v.18, p.981-985, 2004.
SIQUEIRA, J.O.; FRANCO, A.A. Micorrizas. In: SIQUEIRA, J.O.; FRANCO, A.A. (Ed). Biotecnologia do Solo: fundamentos e perspectivas. Brasília, 1988, p.125-177.

SIQUEIRA, J.O.; LAMBAIS, M.R.; STÜRMER, S.L. Fungos Micorrízicos Arbusculares. Biotecnologia Ciência e Desenvolvimento, v.25, p.12-21, 2002.

TRISTÃO, F.S.M.; ANDRADE, S.A.L. DE; SILVEIRA, A.P.D. DA. Fungos micorrízicos arbusculares na formaçáo de mudas de cafeeiro, em substratos orgânicos comerciais. Bragantia, v.65, p.649-658, 2006.

VANDRESEN, J.; NISHIDATE, F.R.; TOREZAN, J.M.D.; ZANGARA, W. Inoculação de fungos micorrízicos arbusculares e adubação na formação e pós-transplante de mudas de cinco espécies arbóreas nativas do sul do Brasil. Acta Botânica Brasilica, v.21, p.753-765, 2007.

WANG, B.; QIU, Y-L. Phylogenetic distribution and evolution of mycorrhizas in land plants. Mycorrhiza, v.16, p.299-363, 2006. 